\title{
Feasibility and outcomes of the removable prosthesis in medically compromised patients
}

\author{
Abdullah F. Al-Hussain ${ }^{1 *}$, Yahya S. Aljabri' ${ }^{2}$ Khalid A. Sindi ${ }^{3}$, Abdulaziz Y. Thobab', \\ Abdullah A. Khormi ${ }^{4}$, Faisal A. Daghreeri ${ }^{2}$, Raghad M. Bediwi ${ }^{3}$, Fawaz S. Alolaywi ${ }^{5}$, \\ Nadia H. Habbal ${ }^{6}$, Shoroq M. AlQahtani ${ }^{7}$, Sana F. Alkhamis ${ }^{8}$
}

\author{
${ }^{1}$ Department of Advanced General Dentistry, Ministry of National Guards Health Affairs, Riyadh, Saudi Arabia \\ ${ }^{2}$ College of Dentistry, Jazan University, Jazan, Saudi Arabia \\ ${ }^{3}$ Department of General Dentistry, King Abdulaziz University, Jeddah, Saudi Arabia \\ ${ }^{4}$ Department of General Dentistry, Ministry of Health, Jazan, Saudi Arabia \\ ${ }^{5}$ Department of General Dentistry, King Saud Hospital, Al-Qassim, Saudi Arabia \\ ${ }^{6}$ Department of General Dentistry, Andalusia Dental Centers, Jeddah, Saudi Arabia \\ ${ }^{7}$ College of Dentistry, King Khalid University, Abha, Saudi Arabia \\ ${ }^{8}$ College of Dentistry, Majmaah University, Zulfi, Saudi Arabia
}

Received: 26 August 2021

Accepted: 01 September 2021

*Correspondence:

Dr. Abdullah Fahad Al-Hussain,

E-mail: dr.abdullahalhussain@gmail.com

Copyright: (c) the author(s), publisher and licensee Medip Academy. This is an open-access article distributed under the terms of the Creative Commons Attribution Non-Commercial License, which permits unrestricted non-commercial use, distribution, and reproduction in any medium, provided the original work is properly cited.

\begin{abstract}
Increasing awareness about providing the necessary care of oral health is essential for patients with a removable prosthesis. The awareness is less interested in dental disorders, being busier with the systemic ones. Planning prosthetic management should only be done after a thorough evaluation of the underlying systemic conditions because the treatment plan might vary accordingly. Previous studies have demonstrated that many systemic diseases are associated with removable prosthesis (RP) procedures. We aim to discuss the feasibility and outcomes of the removable prosthesis for medically compromised patients, including many conditions as diabetes mellitus, cardiovascular, renal, hematological, pulmonary, gastrointestinal disorders and immunocompromised patients. Taking adequate care of oral hygiene practices is recommended for these patients. In addition, it is necessary to have frequent care, but short followup visits to make sure that the modalities are still valid for additional use, and to investigate for potential complications and adverse events. Adequate prophylaxis against some diseases might also be the only convenient solution. Therefore, proper management of the medical condition might be associated with the enhanced success of the modality with favorable outcomes and better quality of life. Making unified protocols to deal with these patients is also suggested to enhance the practice among the different clinical settings.
\end{abstract}

Keywords: Removable prosthesis, Dentistry, Systemic disorders, Management

\section{INTRODUCTION}

The systemic health among dental patients is hugely variable, and estimates show that the prevalence of some systemic disorders that might interfere with the dental management modalities is high across the different global populations. Research also shows that a high percentage of these patients requires the integration of increased prosthetic care, as it has been shown that around $25 \%$ of the 35-74-year aged populations are edentulous. ${ }^{1}$ It is becoming more evident that systemic diseases are in a significant correlation with oral health. Screening and evaluation of the systemic conditions are becoming an essential part of the dental settings. Therefore, adequate 
efforts should be directed to the management of these diseases to achieve better dental outcomes. Increasing awareness about providing the necessary care of oral health is essential for these patients that are less interested in dental disorders, being busier with the systemic ones. Furthermore, planning prosthetic management should only be done after a thorough evaluation of the underlying systemic conditions because the treatment plan might vary accordingly. ${ }^{2,3}$ Previous studies have demonstrated that many systemic diseases are associated with removable prosthesis (RP) procedures. ${ }^{4,5}$ In the present study, We aim to discuss the feasibility and outcomes of the removable prosthesis for medically compromised patients, including many conditions as diabetes mellitus, cardiovascular, renal, hematological, pulmonary, gastrointestinal disorders and immunocompromised patients.

\section{METHODS}

This literature review is based on an extensive literature search in Medline, Cochrane, and EMBASE databases which was performed on $25^{\text {th }}$ July 2021 using the medical subject headings $(\mathrm{MeSH})$ or a combination of all possible related terms. This was followed by the manual search for papers in Google Scholar while the reference lists of the initially included papers. ${ }^{6,7}$ Papers discussing the feasibility and outcomes of the removable prosthesis were screened for relevant information, with no limitation placed on date, language, age of participants, or publication type.

\section{DISCUSSION}

In general, the global prevalence of systemic and dental diseases is variable across different countries and populations. Estimates show that the prevalence of chronic kidney disease is $10.6-13.4 \%$, the prevalence of diabetes is $8.5 \%$, the prevalence of leukemia is $2.5 \%$, the prevalence of anemia is $24.8 \%$, while the prevalence of hyperthyroidism is $0.2-1.3 \% .^{8-12}$ Other estimates also indicate that around 43.8 million patients suffer from dementia, while 422.7 million patients suffer from cardiovascular diseases globally. ${ }^{13,14}$ Besides, it has been estimated that dental diseases are the most common globally, with an estimate that 5 billion people suffer from dental decays. ${ }^{15}$ Furthermore, previous investigations have demonstrated variable rates for the prevalence of systemic diseases among patients suffering from dental disorders. A previous study demonstrated that around two-thirds of dental patients had a previous history of at least one medical condition, while half of the dental patients were also reported to suffer from multiple medical conditions. ${ }^{16}$ Another investigation also reported that $35.2 \%$ of their included population with systemic diseases required dental consultation within a public setting, while only $28.1 \%$ were reported for private settings. ${ }^{17}$ Accordingly, the following section will be divided into the commonest medical conditions that are associated with RP to discuss the feasibility and outcomes of these modalities in these patients.

\section{Diabetes mellitus}

Reports show that edentulism is significantly associated with diabetes mellitus, and both types of the disease have been reported to increase the risk of periodontal diseases and denture stomatitis. ${ }^{18,19}$ The complications and adverse events of diabetes mellitus are various, and many of these have been reported within the oral cavity. Moreover, some of these complications might include loss of the alveolar bones, aggressive periodontal diseases, increased risk of formation of periodontal abscesses, candidiasis, xerostomia, and denture stomatitis. Furthermore, it has been reported that the disease is also associated with an increased risk of resorption of the residual ridge. ${ }^{20}$ Accordingly, using minimal pressure techniques is recommended for these patients to potentially record the impression for further implications. Besides, in cases suffering from severe xerostomia, it is recommended to care for a salivary denture reservoir. Hyperglycemia has been associated with many oral adverse events, including candidiasis and the development of many oral pathogens that can induce serious problems. Besides, previous studies indicate the efficacy of oral hypoglycemic drugs administration on eradicating oral candidiasis in patients with dental wear. ${ }^{21}$ Adequate control of the blood glucose levels using oral hypoglycemic modalities. Therefore, enhance the quality of oral care and health and be associated with better outcomes in diabetic patients with dental wear. ${ }^{21}$ Accordingly, advising diabetic patients to adequately administer their oral anti-hyperglycemic modalities and continuously brushing their teeth is mandatory to achieve better prevention and enhance the outcomes.

Moreover, adequate education for the patients about their oral health is also recommended to urge these patients to maintain adequate oral health and hygiene, with the frequent use of denture cleaning modalities. Caring for the structure of the intended RP modality is also important, and it has been recommended that such modalities should be with wide interdental, self-cleansing spaces, not covered by marginal gingiva, with embrasures regions, with maximum retention and free gliding occlusions, and with a specific contact point between the natural abutment teeth and the denture. It is now widely accepted to say that the masticatory functions are poorer with RP modalities than the fixed and implant prosthesis. ${ }^{22}$ Accordingly, previous studies have recommended that implant treatment should be done using the least traumatic approaches within a stress-free environment to enhance the outcomes and make these modalities feasible when diabetes is adequately controlled. ${ }^{22,23}$ Increased risk of inflammation and infection might also affect the prognosis in these patients. Therefore, frequent follow-up observations are recommended.

\section{Cardiovascular, renal, and hematological diseases}

There is no strong evidence about an existing association between cardiovascular diseases and removable dentures. 
However, it has been previously demonstrated that the quantity (number), and quality (maintainance) of the remaining teeth, in addition to removing any potentially observed inflammatory foci are good predictors for survival among patients with cardiovascular diseases. ${ }^{24} \mathrm{~A}$ previous investigation by Ercalik-Yalcinkaya et al reported that among their 400 included subjects with dental wear, hypertension was the most commonly diagnosed disorder among them. ${ }^{25}$ For denture wear patients, there is also an increased risk of periodontitis and denture stomatitis, which can furtherly disseminate into the patient's blood, leading to the potential development of many chronic systemic diseases, including stroke, atherosclerosis, and hypertension. ${ }^{26-28}$ The increased risk of dissemination from the dental infections might be mainly attributable to dental wounds and manipulation. Therefore, adequately taking care of these events might significantly enhance the prognosis. Besides, prophylactic drugs might also be indicated in such cases to prevent any potential risk of dissemination and the development of systemic diseases. Dentists should, therefore, advise their patients to maintain adequate oral health and hygiene measures to achieve better outcomes. ${ }^{24,29}$

In general, patients suffering from cardiovascular diseases should be given short appointments and the surgical procedures should be conducted according to properly planned stress reduction protocols. These patients usually suffer from reduced blood to the peripheral tissues and reduced oxygenation. Therefore, using local vasoconstrictors is not recommended. Furthermore, gingival hyperplasia might also be associated as a result of the administration of calcium-channel blockers, as antihypertensive drugs. Consequently, replacing these modalities might be needed in some cases. Although anemic patients might be subjected to an increase the risk of impaired bone development and maturation, and implants can still be used. However, this requires the administration of certain prophylactic protocols such as RP. On the other hand, this might be a suitable option for these patients with minimal risk of adverse events and enhanced outcomes. Among patients with leukemia, removal prostheses can be used. However, these should not irritate the underlying soft tissues, and patients are advised to maintain good oral health to enhance the prognosis and outcomes. Nevertheless, it should be noted that fixed partial dentures are the most commonly recommended modalities for these patients that have been associated with reduced risk of injury and complications to the gingival tissues. Among patients suffering from renal diseases, caring for dental problems should be conducted before initiating renal transplantation because the process requires the administration of immunosuppressive modalities that might flare up systemic infections that are usually associated with invasive dental procedures. The management of the dental disorders in these patients should be conducted by non-surgical procedures, including medications or laser therapy. Accordingly, dental implants might be contraindicated for some patients. On the other hand, RP can be indicated in such cases with minimally invasive modalities.

\section{Pulmonary diseases and Helicobacter pylori infections}

Respiratory infections with Candida pneumonia are rare. However, many studies have demonstrated that oral bacteria are responsible for many respiratory infections. ${ }^{30,31}$ Moreover, it was previously demonstrated that these oral pathogens might colonize dental plaque that might develop following the administration of the RP modalities. ${ }^{32}$ It has been demonstrated that the continuous use of RP during sleep might be associated with an increased risk of inflammation, infections, and pneumonia. ${ }^{33}$ Accordingly, routine and continuous caring of oral health might be associated with a significant reduction in these burdens to enhancing the related outcomes in the elderly population. In this context, a previous investigation in Japan reported that reduced frequencies of febrile illness, pneumonia, and pneumoniainduced mortality over a 2-year follow-up duration were significantly associated with the continuous care of oral health and oral hygiene for the 400 study participants that were wearing dentures. ${ }^{34}$ Accordingly, it has been suggested that performing oral hygiene measures and protocols should be adequately conducted by the healthcare givers to prevent pneumonia and other associated complications. ${ }^{35}$ The risk of pneumonia is in direct association with the status of bacterial colonization among patients that wear dentures. ${ }^{32,36}$ Besides, it has been demonstrated that vulnerable patients might significantly benefit from the continuous care of their oral health. ${ }^{36}$ Besides, patients suffering from chronic obstructive pulmonary diseases have been shown to have significant amounts of bacterial colonizations within their lower respiratory tracts during all the stages of the disease, which was attributable to wearing RP. Furthermore, the incidence of mucosal infections and prosthetic stomatitis is higher in these patients as compared to the healthy controls. ${ }^{37}$ In cases of apparent inflammation that is induced by denture wear. Furthermore, there is an increased risk of developing superficial candidiasis. ${ }^{38}$ Swarming pulmonary circulation, which might result from bacterial colonization from the continuous use of RP, can also impact the esophageal air channels, leading to further complications and adverse events. $^{39}$

Gastric inflammation might be associated with inflammation and infection to the oral mucosa that is usually caused by fungal or bacterial pathogens. In $100 \%$ of patients suffering from Helicobacter pylori-induced gastritis, oral infections were associated, indicating a strong correlation between the pathogen and oral health. Nonetheless, the mechanism is not still fully comprehended. ${ }^{40}$ Adequate management of Helicobacter pylori infection that resides in the oral cavity is a difficult process, as it has been noticed that even after eradicating the pathogen from the oral cavity by antibiotics. It can still infect the stomach within few weeks after applying the management modalities. ${ }^{41}$ Besides, it has been reported 
that even with adequate oral hygiene practices, adequate management of the pathogen is still difficult to achieve. ${ }^{42}$ On the other hand, other researchers have demonstrated that adequately managing dental plaques might effectively facilitate the process of eradication of Helicobacter pylori bacteria. ${ }^{43}$ Moreover, re-infection, relapse, and gastric ulcers might also be significantly correlated with the previous infection, even after adequately eradicating the bacteria. ${ }^{44}$ Therefore, these patients should be admitted to suitable prophylactic programs that can effectively intervene against such issues and enhance the associated outcomes.

\section{Others}

The entrapment of microorganisms has become easier as a result of the surface irregularities of the dentures, which increases the risk of bacterial colonization and infections. Accordingly, immunosuppressed patients, by iatrogenic causes, following human immunodeficiency virusinfections, or due to other causes, are not recommended to use RP because of the increased potential complications from the increased frequency of infections. ${ }^{45}$ Evidence from the literature shows that these patients have higher frequencies of bacterial isolates from their oral mucosa, which is mainly attributable to wearing RPs. It has been demonstrated that wearing RPs is significantly associated with the increased incidence of candidiasis among patients suffering from HIV infections, and the levels of CD4 were also reported to be in a significant correlation with the development of oral candidiasis. ${ }^{46}$ Daniluk et al previously reported that the rate of candidiasis was higher among patients with dentures than other patients without it. ${ }^{47}$ The incidence of this infection increases with multiple factors as chemotherapy or radiotherapy, and with patients that frequently administer immunosuppressant drugs or antibiotics. ${ }^{48}$ Cancer patients might also suffer from an immunocompromised state. As a result, the rates of infections might also be high in these patients. Moreover, evidence shows that Gram-negative Enteric bacilli and Staphylococcus spp. were effectively isolated from the palate, tongue, and dental plaques in a more frequent pattern in cancer patients than other patients without cancers. ${ }^{47}$ The presence of history with inflammatory conditions might precede the presence of a neoplasm, while in other cases, inflammation comes secondary to the presence of a neoplasm, and therefore, adequate oral hygiene practices are recommended to enhance the associated outcomes. ${ }^{30,49,50}$.

\section{CONCLUSION}

Taking adequate care of oral hygiene practices is recommended for these patients. In addition, it is necessary to have frequent care, but short follow-up visits to make sure that the modalities are still valid for additional use, and to investigate for potential complications and adverse events. Adequate prophylaxis against some diseases might also be the only convenient solution. Therefore, proper management of the medical condition might be associated with the enhanced success of the modality with favorable outcomes and better quality of life. Making unified protocols to deal with these patients is also suggested to enhance the practice among the different clinical settings.

Funding: No funding sources

Conflict of interest: None declared

Ethical approval: Not required

\section{REFERENCES}

1. Agerberg G, Carlsson G. Chewing Ability in Relation to Dental and General Health. Acta Odontologica Scandinavica. 1981;39:147-53.

2. Suwal P, Pk P, Head, Professor A. General Systemic Evaluation of Prosthodontic Patients: A Literature Review. Journal of Nepal Dental Association. 2013;13:90-4.

3. Le Bars P, Kouadio AA, N'Goran JK, Badran Z, Soueidan A. Relationship between removable prosthesis and some systemics disorders. J Indian Prosthodont Soc. 2015;15(4):292-9.

4. Dietrich T, Garcia RI. Associations between periodontal disease and systemic disease: evaluating the strength of the evidence. Journal of periodontology. 2005;76(11):2175-84.

5. Senpuku H, Sogame A, Inoshita E, Tsuha Y, Miyazaki H, Hanada N. Systemic diseases in association with microbial species in oral biofilm from elderly requiring care. Gerontology. 2003;49(5):301-9.

6. Hashan MR, Ghozy S, El-Qushayri AE, Pial RH, Hossain MA, Al Kibria GM. Association of dengue disease severity and blood group: A systematic review and meta-analysis. Rev Med Virol. 2021;31(1):1-9.

7. El-Qushayri AE, Ghozy S, Abbas AS, et al. Hyperimmunoglobulin therapy for the prevention and treatment of congenital cytomegalovirus: a systematic review and meta-analysis. Expert Rev Anti Infect Ther. 2020:1-9.

8. Hill NR, Fatoba ST, Oke JL. Global Prevalence of Chronic Kidney Disease - A Systematic Review and Meta-Analysis. PloS one. 2016;11(7):e0158765.

9. Ginter E, Simko V. Global prevalence and future of diabetes mellitus. In: Diabetes. Springer; 2013:35-41.

10. De Benoist B, Cogswell M, Egli I, McLean E. Worldwide prevalence of anaemia 1993-2005; WHO global database of anaemia. 2008.

11. Mohammadian M, Pakzad R, MohammadianHafshejani A, Salehiniya H. A study on the incidence and mortality of leukemia and their association with the human development index (HDI) worldwide in 2012. 2018.

12. Taylor PN, Albrecht D, Scholz A, et al. Global epidemiology of hyperthyroidism and hypothyroidism. Nature reviews Endocrinology. 2018;14(5):301-16.

13. Roth GA, Johnson C, Abajobir A. Global, Regional, and National Burden of Cardiovascular Diseases for 
10 Causes, 1990 to 2015. Journal of the American College of Cardiology. 2017;70(1):1-25.

14. Global, regional, and national burden of Alzheimer's disease and other dementias, 1990-2016: a systematic analysis for the Global Burden of Disease Study 2016. The Lancet Neurology. 2019;18(1):88-106.

15. Petersen PE. The World Oral Health Report 2003: continuous improvement of oral health in the $21 \mathrm{st}$ century--the approach of the WHO Global Oral Health Programme. Community dentistry and oral epidemiology. 2003;31:3-23.

16. Cottone JA, Kafrawy AH. Medications and health histories: a survey of 4,365 dental patients. Journal of the American Dental Association (1939). 1979;98(5):713-8.

17. Fernández Feijoo J, Garea-Gorís R, FernándezVarela M, Tomás Carmona I, Diniz-Freitas M, Limeres J. Prevalence of systemic diseases among patients requesting dental consultation in the public and private systems. Medicina oral, patologia oral y cirugia bucal. 2011;17:89-93.

18. Felton DA. Edentulism and comorbid factors. Journal of prosthodontics : official journal of the American College of Prosthodontists. 2009;18(2):88-96.

19. Phelan JA, Levin SM. A prevalence study of denture stomatitis in subjects with diabetes mellitus or elevated plasma glucose levels. Oral surgery, oral medicine, and oral pathology. 1986;62(3):303-5.

20. Al-Jabrah O. Association of type 2 diabetes mellitus with the reduction of mandibular residual ridge among edentulous patients using panoramic radiographs. Open Journal of Stomatology. 2011;1.

21. Ganapathy DM, Joseph S, Ariga P, Selvaraj A. Evaluation of the influence of blood glucose level on oral candidal colonization in complete denture wearers with Type-II Diabetes Mellitus: An in vivo Study. Dental research journal. 2013;10(1):87-92.

22. Lee JH, Han JS, Han K, Lee SY. Association between Diabetes and the Use of Removable Dental Prostheses among the Korean Population. Journal of Korean medical science. 2019;34(41):262.

23. Kapur KK, Garrett NR, Hamada MO. A randomized clinical trial comparing the efficacy of mandibular implant-supported overdentures and conventional dentures in diabetic patients. Part I: Methodology and clinical outcomes. The Journal of prosthetic dentistry. 1998;79(5):555-69.

24. Janket SJ, Surakka M, Jones JA. Removable dental prostheses and cardiovascular survival: a 15-year follow-up study. Journal of dentistry. 2013;41(8):740-6.

25. Ercalik-Yalcinkaya S, Özcan M. Association between Oral Mucosal Lesions and Hygiene Habits in a Population of Removable Prosthesis Wearers. Journal of prosthodontics: official journal of the American College of Prosthodontists. 2015;24(4):271-8.

26. Gendreau L, Loewy ZG. Epidemiology and etiology of denture stomatitis. Journal of prosthodontics : official journal of the American College of Prosthodontists. 2011;20(4):251-60.

27. Demmer RT, Desvarieux M. Periodontal infections and cardiovascular disease: the heart of the matter. Journal of the American Dental Association (1939). 2006;137:14-20.

28. Scannapieco FA, Genco RJ. Association of periodontal infections with atherosclerotic and pulmonary diseases. Journal of periodontal research. 1999;34(7):340-5.

29. Corsalini M, Rapone B, Grassi FR, Di Venere D. A study on oral rehabilitation in stroke patients: analysis of a group of 33 patients. Gerodontology. 2010;27(3):178-82.

30. Green SL. Anaerobic pleuro-pulmonary infections. Postgraduate medicine. 1979;65(1):62-6.

31. Martin BJ, Corlew MM, Wood $\mathrm{H}$, et al. The association of swallowing dysfunction and aspiration pneumonia. Dysphagia. 1994;9(1):1-6.

32. Sumi Y, Miura H, Sunakawa M, Michiwaki Y, Sakagami N. Colonization of denture plaque by respiratory pathogens in dependent elderly. Gerodontology. 2002;19(1):25-29.

33. Iinuma T, Arai Y, Abe Y. Denture wearing during sleep doubles the risk of pneumonia in the very elderly. Journal of dental research. 2015;94(3):28-36.

34. Yoneyama T, Yoshida M, Ohrui T. Oral care reduces pneumonia in older patients in nursing homes. Journal of the American Geriatrics Society. 2002;50(3):430-3.

35. El-Solh AA. Association between pneumonia and oral care in nursing home residents. Lung. 2011;189(3):173-80.

36. Scannapieco FA. Pneumonia in nonambulatory patients. The role of oral bacteria and oral hygiene. Journal of the American Dental Association (1939). 2006;137:21-5.

37. Przybyłowska D, Mierzwińska-Nastalska E, Rubinsztajn R, Chazan R, Rolski D, Swoboda-Kopeć E. Influence of denture plaque biofilm on oral mucosal membrane in patients with chronic obstructive pulmonary disease. Advances in experimental medicine and biology. 2015;839:25-30.

38. Gümrü B, Kadir T, Uygun-Can B, Ozbayrak S. Distribution and phospholipase activity of Candida species in different denture stomatitis types. Mycopathologia. 2006;162(6):389-94.

39. Gasparoto TH, de Oliveira CE, Vieira NA. Activation pattern of neutrophils from blood of elderly individuals with Candida-related denture stomatitis. European journal of clinical microbiology \& infectious diseases : official publication of the European Society of Clinical Microbiology. 2012;31(6):1271-7.

40. Loster BW, Majewski SW, Cześnikiewicz-Guzik M, Bielanski W, Pierzchalski P, Konturek SJ. The relationship between the presence of Helicobacter pylori in the oral cavity and gastric in the stomach. Journal of physiology and pharmacology : an official 
journal of the Polish Physiological Society. 2006;57:91-100.

41. Thieu H, Bach Dat B, Nam NH. Antibiotic resistance of Helicobacter pylori infection in a children's hospital in Vietnam: prevalence and associated factors. Minerva medica. 2020;111(5):498-501.

42. Namiot DB, Namiot Z, Kemona A, Bucki R, Gotebiewska M. Oral health status and oral hygiene practices of patients with peptic ulcer and how these affect Helicobacter pylori eradication from the stomach. Helicobacter. 2007;12(1):63-7.

43. Jia CL, Jiang GS, Li CH, Li CR. Effect of dental plaque control on infection of Helicobacter pylori in gastric mucosa. Texas dental journal. 2012;129(10):1069-73.

44. Berroteran A, Perrone M, Correnti M. Detection of Helicobacter pylori DNA in the oral cavity and gastroduodenal system of a Venezuelan population. Journal of medical microbiology. 2002;51(9):764-70.

45. Perezous LF, Flaitz CM, Goldschmidt ME, Engelmeier RL. Colonization of Candida species in denture wearers with emphasis on HIV infection: a literature review. The Journal of prosthetic dentistry. 2005;93(3):288-93.

46. Witzel AL, Pires Mde F, de Carli ML, Rabelo GD, Nunes TB, da Silveira FR. Candida albicans isolation from buccal mucosa of patients with HIV wearing removable dental prostheses. The International journal of prosthodontics. 2012;25(2):127-31.

47. Daniluk T, Fiedoruk K, Sciepuk M,. Aerobic bacteria in the oral cavity of patients with removable dentures. Advances in medical sciences. 2006;51:86-90.

48. Samaranayake LP, Hughes A, Weetman DA, MacFarlane TW. Growth and acid production of Candida species in human saliva supplemented with glucose. Journal of oral pathology. 1986;15(5):2514.

49. Lawrence M, Aleid W, McKechnie A. Access to dental services for head and neck cancer patients. The British journal of oral \& maxillofacial surgery. 2013;51(5):404-7.

50. Meurman JH, Grönroos L. Oral and dental health care of oral cancer patients: hyposalivation, caries and infections. Oral oncology. 2010;46(6):464-7.

Cite this article as: Hussain AF, Aljabri YS, Sindi KA, Thobab AY, Khormi AA, Daghreeri FA, et al. Feasibility and outcomes of the removable prosthesis in medically compromised patients. Int J Community Med Public Health 2021;8:5142-7. 